\title{
Metabolomics Applications in Plant Biotechnology
}

\section{Diego F. Gomez-Casati*}

Center of Photosynthetic and Biochemical Studies (CEFOBI-CONICET), National University of Rosario, Suipacha 531, 2000 Rosario, Argentine

Metabolomics, the comprehensive analysis in which all the metabolites of an organism are identified and quantified, has recently emerged as a functional genomics methodology that contributes to the knowledge of the many complex molecular interactions in different biological systems [1]. Thus, the study of a metabolome represents the logical progression from large-scale analysis of RNA and proteins at the systems level [2-4].

Currently, there are many metabolomics applications in different areas for example Medicine, Systems Biology, Food technology and Microbial and Plant Biotechnology, among others.

Metabolites found in plants are involved in many functions such as development, growth, germination, aroma and scent of flowers, taste of fruits, stress responses and pathogen resistance. Thus, one of the aims of metabolomics is to determine variations in the concentration of metabolites in different metabolic networks and relate these changes with the phenotype, physiological and/or developmental state of a cell, tissue or organism [4].

Particularly, the metabolic profile of plant organisms is highly complex due to the wide chemical diversity and the variable range of concentration (from $\mathrm{pM}$ to $\mathrm{mM}$ ) of metabolites. It has been reported in plants more than 200,000 metabolites, many of which have a specific role in adapting to different situations such as temperature changes or stress conditions, among others [4].

As mentioned above, plant metabolomics is being progressively used for the study of the differential response to nutrient uptake and stress conditions. In addition, other metabolomics applications are the engineering of various metabolic pathways existing in an organisms, as well as the engineering of new biochemical pathways, including those that improve nutrient utilization [5].

In the recent past few years, many efforts were made to avoid pathogen attack to plant cultures. Different pathogens, such as fungi, bacteria, and viruses has a negative impact on crop production. To prevent this, plants had developed some strategies such as the alteration of gene expression, activation of key metabolic pathways and posttranslational modification of proteins. The mechanisms mentioned above ultimately produce the increment of primary and secondary metabolites involved in defense response of plants to pathogenic organisms [4].

Taking into account the recent advances in the study of different omic's techniques, it has been possible to progress in the characterization of plants transcriptomes, proteomes and metabolomes during pathogen attack, and the modulation of the response after the infection. This approach becomes extremely important to know in detail the mechanisms used by diseases-causing agents to infect plants and thus, to develop different strategies to produce plant organisms with increased resistance to pathogens [6].

The ultimate goal of plant metabolomics is to understand and predict, in conjunction with other omic's sciences the function of genes and proteins involved in a particular biochemical pathway and to better know the behavior of complex systems such as higher plants by using the results obtained from data mining tools for subsequent modeling and simulation.

\section{References}

1. Hall R, Beale M, Fiehn O, Hardy N, Sumner L, et al. (2002) Plant metabolomics: the missing link in functional genomics strategies. Plant Cell 14: 1437-1440.

2. Bino RJ, Hall RD, Fiehn O, Kopka J, Saito K, et al. (2004) Potential of metabolomics as a functional genomics tool. Trends Plant Sci 9: 418-425.

3. Weckwerth W (2003) Metabolomics in systems biology. Annu Rev Plant Biol 54: 669-689.

4. Gomez-Casati DF, Zanor MI, Busi MV (2013) Metabolomics in plants and humans: applications in the prevention and diagnosis of diseases. Biomed Res Int 2013: 792527.

5. Bhalla R, Narasimhan K, Swarup S (2005) Metabolomics and its role in understanding cellular responses in plants. Plant Cell Rep 24: 562-571.

6. Gomez-Casati DF, Pagani MA, Busi MV, Bhadauria V (2016) Omics Approaches for the Engineering of Pathogen Resistant Plants. Curr Issues Mol Biol 19: 89-98.
${ }^{*}$ Corresponding author: Diego F. Gomez-Casati, Center of Photosynthetic and Biochemical Studies (CEFOBI-CONICET), National University of Rosario, Suipacha 531, 2000 Rosario, Argentine, Tel: 543414371955; E-mail: gomezcasati@cefobiconicet.gov.ar

Received April 29, 2016; Accepted May 05, 2016; Published May 07, 2016

Citation: Gomez-Casati DF (2016) Metabolomics Applications in Plant Biotechnology. Metabolomics 6: e146. doi:10.4172/2153-0769.1000e146

Copyright: (c) 2016 Gomez-Casati DF. This is an open-access article distributed under the terms of the Creative Commons Attribution License, which permits unrestricted use, distribution, and reproduction in any medium, provided the original author and source are credited. 